\title{
DENGUE CASES REPORTED DURING 2015-17 IN A TERTIARY CARE HOSPITAL OF ISLAMABAD
}

\author{
Abdul Wali Khan ${ }^{1}$, Shahid Hanif ${ }^{2}$, Shagufta Zafar Qureshi ${ }^{3}$, Zakir Hussain $^{4}$
}

${ }^{1-4}$ Federal Government Polyclinic Hospital Islamabad

Correspondence:Abdul Wali Khan, Email: khanzadaamc@yahoo.com

\begin{abstract}
Background: Dengue has emerged as a vector-borne disease with an increase in number of cases with multiple outbreaks throughout Pakistan and hospitalization each passing year, thus stretching available health resources in Pakistan.

Methods: This was a descriptive cross-sectional study involving 233 dengue fever indoor cases. Complete clinical findings were recorded followed by laboratory and other diagnostic tests.

Results: Most of the cases were admitted from peri-urban areas of Islamabad with symptoms of fever. Seasonal patterns of typical post-monsoon and clusters of cases from low socio-economic backgrounds from areas of Bhara Kau, Bari-Imam, Sohan and G6 and G-7 sectors were found. Platelets and WBCs counts were found on the lower side of the normal values. IgM and IgG and NS1 tests were performed. Affected age group) was young (20-40 years) and male gender being more exposed to vector.

Conclusion: Dengue has emerged in the poor peri-urban areas of Islamabad typically in post-monsoon seasons reaching peak during the months of September, gradually declining and waning off in the month of December. A combination of clinical symptoms and diagnostic tests IgM, IgG and NS1 could greatly help in early diagnosis and treatment of cases thus minimizing compilations and mortality.

Key words: Dengue, hospital surveillance, ns1, vector aedes agypti, igm, igg
\end{abstract}

\section{Introduction}

Dengue is a vector-borne viral disease affecting 300-500 million people globally in more than 128 countries, making half of the world-population at risk of getting the infection $(1,2)$. Due to lack of availability of an effective vaccine and proven anti-viral specific medications, dengue has emerged a public health challenge and an enormous burden on available resources $(3,4)$. In Pakistan, dengue has emerged and is rapidly spreading to the non-endemic areas resulting in an increase in hospitalization due to complications. Previous studies in Pakistan have mostly focused on outbreaks and prevalence while very little in Pakistan is known about the linkage of socio-demographic determinants, clinical manifestations and laboratory biomarkers as part of hospital-based surveillance. It is critical to assess dengue cases based on geographical and demographic distribution and as well as by the clinical and laboratory findings simultaneously.

The vector for dengue is a female Aedes aegypti mosquito (5) and also to a lesser extent Aeds albopictus mosquito (6). Causative agent is flavivirus (RNA virus) and the virus has four antigenic distinct serotypes (DEN 1- 4) $(7,8)$. More than $90 \%$ of infection is asymptomatic or having mild self-limiting fever and only in $5 \%$ the infection can lead to severe dengue hemorrhagic fever or further to dengue shock syndrome (9). Complicated cases can result in fatal outcome and death and currently there is a surge in complications in indoor patients (10). Up to $14.9 \%$ of indoor patients need admission in intensive care unit (ICU) and 1.5\% die due to severe complications and delay in treatment (11). Globally more complications and fatalities have been reported in tropical and as well as sub-tropical countries (12).

Dengue's symptoms are usually non-specific and associating fever, aches and fatigue are often present in other endemic infections, thus posing a challenge in differential diagnosis (4). However, a combination of clinical findings and diagnostic tests has proven quite beneficial in timely diagnosis of dengue fever (13). Currently, available antigen NS1 and other antibodies tests (IgM and IgG) have been proven quite useful thus timely supportive and rehydration therapy could greatly reduce mortality in indoor patients $(14,15)$. The aim of this study was to determine socio-demographic determinants and seasonal patterns of dengue based on clinical and laboratory findings in admitted cases for the year 2016 and 2017 in a tertiary care hospital of Islamabad. 


\section{Methodology}

As part of the hospital-based surveillance; data was collected from indoor-patients admitted in Federal Govt. Polyclinic (PGMI) Hospital Islamabad, for the year 2015, 2016 and 2017. Total 233 cases were included in this study. Both male and female patients above the age of 13 years were included. Informed consent was taken prior to data collection. The study was approved by the Ethical Review Committee of Federal Govt. Polyclinic (PGMI), Islamabad. As part of the hospital based surveillance, these cases were admitted in isolation beds reserved for dengue fever cases. Cases were confirmed either on the basis of laboratory or clinical findings. Complete history including the presenting complaints e.g. fever, headache, rash, bleeding etc. were documented. As a part of hospital protocol ; complete blood tests including white blood cells and platelets were performed and these tests were repeated on the next days. Furthermore, Antigen test NS1 and Elisa tests for IgM and IgG were also perfromed. Serum samples for NS1 antigens were taken immediately after admission of suspected or probable dengue fever cases. Dengue Early Rapid (Panbio,USA) was used immediately after admission of patients. This test can identify dengue virus NS1 antigen in serum, plasma or whole blood specimens with a high degree of sensitivity and specificity. Results were interpreted within 15-20 minutes.

Data were entered into spread sheet and later on analyzed in SPSS (IBM SPSS Statistics Version 20). Geographical mapping of the cases from respective areas/union councils was done using Epi-Info (Version 7). P value below 0.05 was considered as significant.

\section{Results}

The most frequent presenting complaints were mostly fever94\% $(n=219 ;)$ of cases. This was accompanied by other symptoms such as headache, body-aches $80 \%$ $(n=200 ;)$, rash and epistaxis. Only $1.3 \%(n=3)()$ subjects had history of previous dengue fever (Table 1)

Blood tests for platelets and WBCs count were performed. Serological tests for $\lg M$ and $\lg G$ antibodies and NS1 for antigens were done. Mean for platelets (N221) and WBCs (N217) was 84679.17 \pm 65513.93 and $6263.65 \pm 9029.20$, respectively. NS1 Antigen test was done in 176 subjects and $89.2 \%(n=157)()$ patients were found positive for NS1. Similarly, IgM was of 92 patients was done and $\operatorname{lgG}$ of 87 cases, which was positive in $66.3 \%$ and $42.5 \%$ cases respectively, table 1 .
Table 1: Demographic data, clinical, laboratory and serological findings among dengue cases, mean $\pm S D$, number (percent)

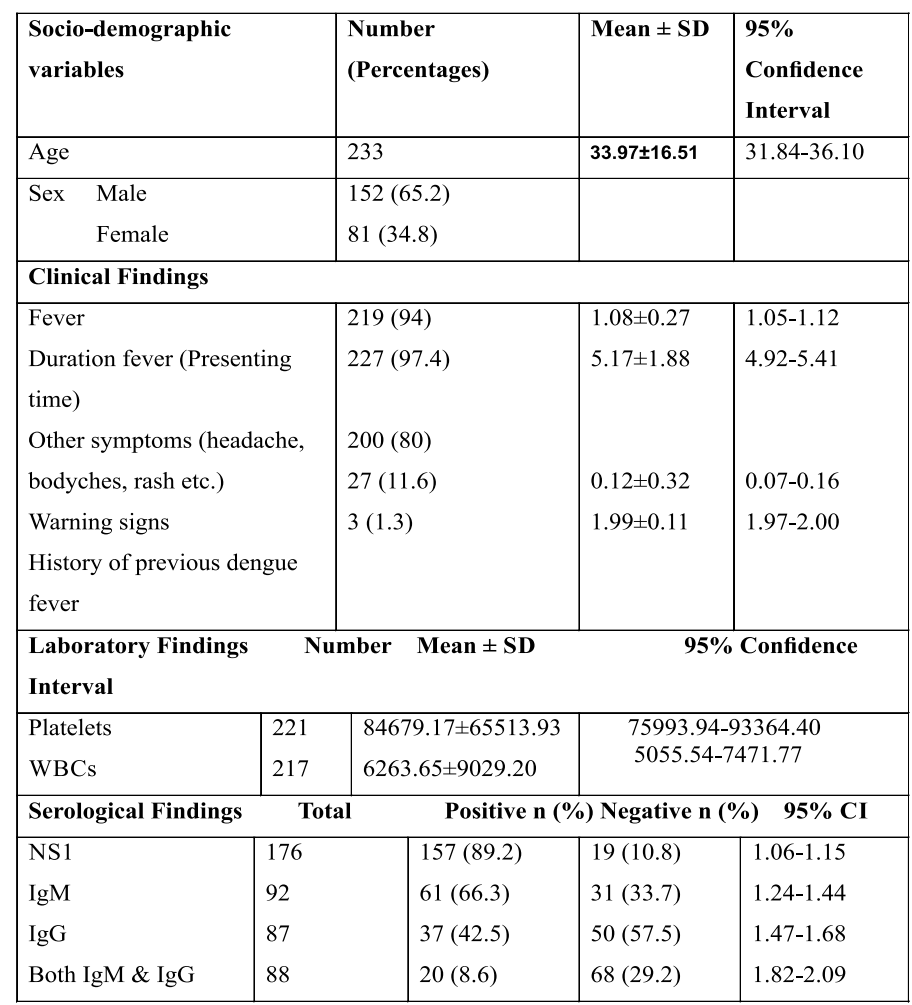

Geographic distribution of the dengue fever cases shows that $25.3 \%)(n=59$; came from Bhara Kahu, Bari Imam 9.9\% $(n=23 ;)$, Sohan 7.7\% $(n=18$; and Sectors G$715 \%(n=35 ;)$ and G-6 10\% (n=24;). During 2016 and 2017 limited outbreaks were reported from Barakau and Sohan (union councils, Tehsils Villages???). From other rural areas of Islamabad $16.3 \%(n=38)()$ cases were admitted. $8.2 \%(n=19)()$ cases came from other areas of District Rawalpindi. These included areas near Faizabad and Iqbal Town areas of Islamabad, which are contiguous to Islamabad District area from where cases were reported (figure 1).

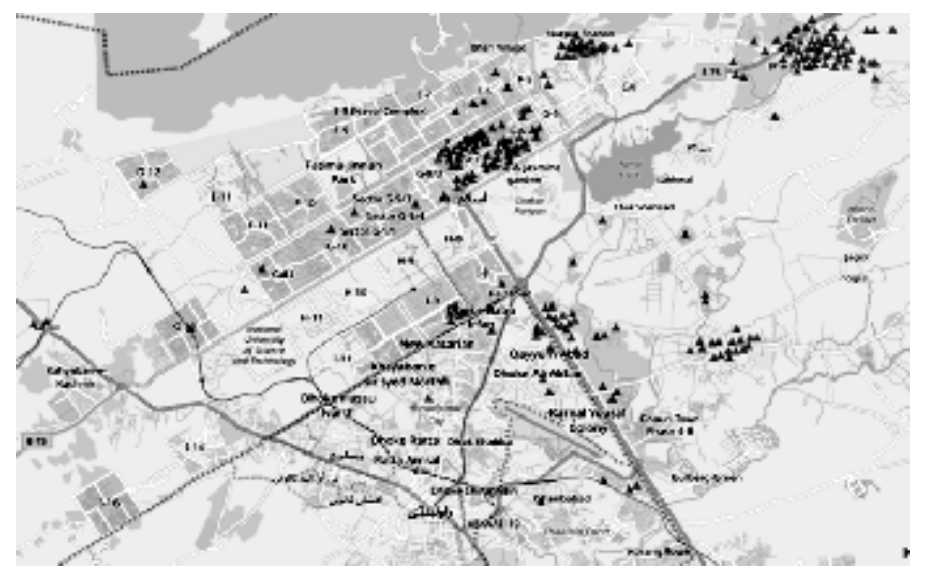

Figure 1: Geographical mapping of cases from areas of Islamabad and adjacent Rawalpindi areas (clusters of cases were reported from Bhara Kau, Bari Imam, Sohan and G-7 and G-7 Sectors 
Patients were admitted in September and the reached peak in the month of October and gradually declined in November and December in each year in 2016 and 2017, respectively (figure 2 ). .

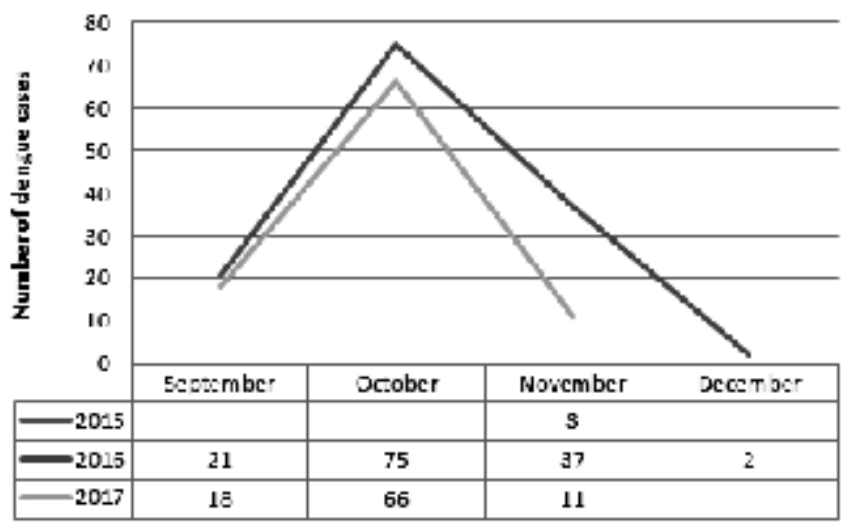

Figure 2: Seasonal patterns of dengue (cases reached at peak in October and then decline in December)

Total number of subjects included in this study were 233 including $65.2 \%(n=152)$ males and $34.8 \%(n=81)$ females. Mean age was $33.97 \pm 16.51$ (Table 1). However, the most affected age group was between 20 40 year ( 119 cases) figure 3 .

\section{Dengue cases based on age group}

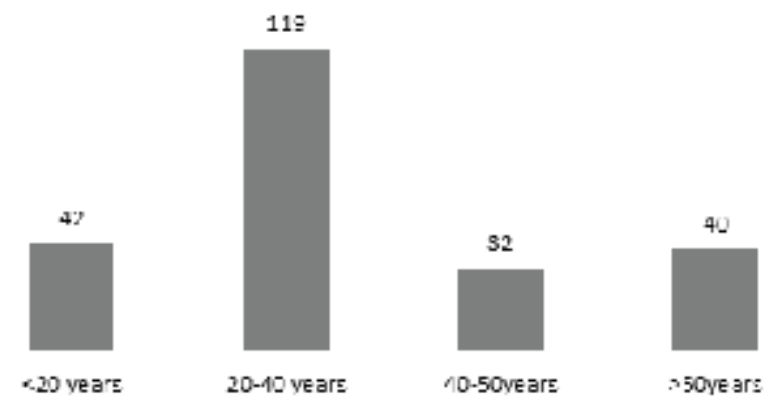

Figure 3: Affected age (more cases reported between $20-40$ year age group)

\section{Discussion}

Mean age of the cases was 33.97 OR 34 and majority of the cases $(65.2 \%)$ were male. The possible explanation is that cases between 20-40 years of age are more engaged in outdoor activities and are more likely to be exposed to the infected vector. The same is for male gender. This is also reported by a study conducted in Pakistan (16), and reported age 32, as peak group and as well as reported affected male gender $61 \%$. Furthermore, in this study it was also observed that with the passage of time, the age group could also affect people having age in second decade and as well as more female gender would be affected by dengue.

Cluster of cases were reported from Bhara Kau, BariImam, Sohan and Sectors G-7 and G-6, as shown in the map. Peri-urban areas and low socioeconomic conditions and as well as lack of proper waste disposal except the Sectors G 7 and 6 could be responsible. Multitude of factors including lack of proper waste disposal, low knowledge, poor background were also reported as complex interplay of factors (17). In Asia (India, Sri Lanka, Myanmar, Thailand, Philippines and Indonesia) in study (18) also addressed eco-bio-social dimensions in urban and peri-urban areas focusing the vector through socio-cultural, local and environmental interventions.

Dengue has been called disease of rainy season (19) and usually outbreaks occur after monsoon (postmonsoon) in Pakistan. Cases are rarely reported before monsoon. In this study the number of cases started in September month for both 2016 and 2017 and it reached its peak in the month of October. It decreased in month of November and December. This is quite typical and consistent with other studies $(16,20,21)$ in this region. The reason is that in this month the humidity and temperature level are optimal for the vector to grow. In Islamabad where the dengue typically cases are reported in these months since September till December (average temperature $27-12{ }^{\circ} \mathrm{C}$ and humidity precipitation $98-37 \mathrm{~mm}$ ). The relative temperature (15$\left.32^{\circ} \mathrm{C}\right)$ and humidity $(30-90$ percent) effects are necessary for spread/tethered flight of Aedes aegypti mosquito, vector for dengue (22). However, in KP cases start from August, reaching peak in September and thus gradually then declining (23). The reason is KP is also the optimal temperature for the vector. Nevertheless, globally areas with suitable temperature and humidity round the year, dengue is present throughout the year (24).

All patients presented typical symptoms of dengue e.g. high grade fever, bodyaches, headache and other symptoms such as rash, epistaxis, gum bleeding etc. Blood tests for platelets and WBCs were found low and were consistent with other studies such as in a study conducted in Karachi (20), where they reported thrombocytopenia $<100,000 / \mathrm{mm} 3$ and in our study the average value was $<84680 / \mathrm{mm} 3$. Here WBCs were also lower than the normal limit. In this study the sensitivity of IgM was $66.3 \%$, which is also higher at earlier stage of disease. Sensitivity of IgG was $42.5 \%$. Sensitivity of both IgM and IgG was $8.6 \%$. In a study conducted in Khyber Pakhtunkhwa (KP) Pakistan in 2011 (23), the combine sensitivity of both IgM and IgG was found to be $3.75 \%$ nearer to our finding. Similarly in the study conducted in $\mathrm{KP}$ the sensitivity of $\operatorname{lgM}$ $(39.35 \%)$ was more than IgG $(22.42 \%)(23)$. IgM was also found in highest proportion of patients in other area e.g. Karachi, Pakistan (16) and as well as in India (25). In FGPC the NS1 was available round the clock. NS1 has been used globally as a sensitive test, in the acute phase of dengue infection and thus this can greatly help in early diagnosis and subsequent treatment and as well as awareness for other subjects (4). NS1 antigenemia is at peak within first 2-4 days of infection and then 
gradually wanes and also diminishes earlier in secondary infection $(26,27)$. Here the sensitivity was $89.2 \%$, which is the range of sensitivity NS1 test, in other developing countries in South America, where it was described to be between $85-96 \%$ (4). However, in a study In Mexico, where the sensitivity of NS1 was found to be $12.3 \%$ (28). In other study (29), the sensitivity was found to be $87 \%$ within first 3 days of fever and then decline to $70 \%$ after 12 days. Thus our findings are compatible with these studies.

\section{Conclusion}

Due to a surge in number of cases in Islamabad and the limited outbreaks from Bhara Kau, Bari-Imam and Sohan areas, the disease could emerge as full outbreak thus affecting the twin cities of Islamabad and Rawalpindi. Awareness, anticipatory approach about the vector (Aedes agypiti) and early diagnosis of cases are warranted.

\section{References}

1. Samuel M, Maoz D, Manrique P, Ward T, RungeRanzinger S, Toledo J, et al. Community effectiveness of indoor spraying as a dengue vector control method: A systematic review. PLoS neglected tropical diseases. 2017;11(8):e0005837.

2. Bhatt S, Gething PW, Brady OJ, Messina JP, Farlow AW, Moyes CL, et al. The global distribution and burden of dengue. Nature. 2013;496(7446):504.

3. Organization WH. Strengthening implementation of the global strategy for dengue fever/dengue haemorrhagic fever prevention and control. Report of the Informal Consultation, 18-20 October 1999, WHO HQ, Geneva, Switzerland. Strengthening implementation of the global strategy for dengue fever/dengue haemorrhagic fever prevention and control Report of the Informal Consultation, 18-20 October 1999, WHO HQ, Geneva, Switzerland. 2000.

4. Pal S, Dauner AL, Mitra I, Forshey BM, Garcia P, Morrison AC, et al. Evaluation of dengue NS1 antigen rapid tests and ELISA kits using clinical samples. PloS one. 2014;9(11):e113411.

5. Rosso F, Pineda JC, Sanz AM, Cedano JA, Caicedo LA. Transmission of dengue virus from deceased donors to solid organ transplant recipients: case report and literature review. The Brazilian Journal of Infectious Diseases. 2018.

6. Stewart-Ibarra AM, Ryan SJ, Kenneson A, King CA, Abbott M, Barbachano-Guerrero A, et al. The burden of dengue fever and chikungunya in southern coastal Ecuador: epidemiology, clinical presentation, and phylogenetics from the first two years of a prospective study. 2018.

7. Craig AT, Joshua CA, Sio AR, Teobasi B, Dofai A, Dalipanda T, et al. Enhanced surveillance during a public health emergency in a resource- limited setting: Experience from a large dengue outbreak in Solomon Islands, 2016-17. PloS one. 2018;13(6):e0198487.

8. Rico-Hesse R. Microevolution and virulence of dengue viruses. Advances in virus research. 2003;59:315.

9. Balmaseda A, Standish K, Mercado JC, Matute JC, Tellez Y, Saborío S, et al. Trends in patterns of dengue transmission over 4 years in a pediatric cohort study in Nicaragua. The Journal of infectious diseases. 2010;201(1):5-14.

10. Vanjare $H$, Mannam $P$, Mishra A, Karuppusami $\mathrm{R}$, Carey R, Abraham A, et al. Brain imaging in cases with positive serology for dengue with neurologic symptoms: a clinicoradiologic correlation. American Journal of Neuroradiology. 2018;39(4):699-703.

11. Yeh C-Y, Chen P-L, Chuang K-T, Shu Y-C, Chien Y-W, Perng GC, et al. Symptoms associated with adverse dengue fever prognoses at the time of reporting in the 2015 dengue outbreak in Taiwan. PLoS neglected tropical diseases. 2017;11(12):e0006091.

12. Gubler DJ. Dengue and dengue hemorrhagic fever. Clinical microbiology reviews. 1998;11(3):480-96.

13. Hermann LL, Thaisomboonsuk B, Poolpanichupatam Y, Jarman RG, Kalayanarooj S, Nisalak A, et al. Evaluation of a dengue NS1 antigen detection assay sensitivity and specificity for the diagnosis of acute dengue virus infection. PLoS neglected tropical diseases. 2014;8(10):e3193.

14. Kalayanarooj S. Standardized clinical management: evidence of reduction of dengue haemorrhagic fever case-fatality rate in Thailand. 1999.

15. Kittigul L, Pitakarnjanakul P, Sujirarat D, Siripanichgon K. The differences of clinical manifestations and laboratory findings in children and adults with dengue virus infection. Journal of Clinical Virology. 2007;39(2):76-81.

16. Khan E, Kisat M, Khan N, Nasir A, Ayub S, Hasan R. Demographic and clinical features of dengue fever in Pakistan from 2003-2007: a retrospective cross-sectional study. PloS one. 2010;5(9):e12505.

17. Arunachalam N, Tana S, Espino F, Kittayapong $\mathrm{P}$, Abeyewickrem W, Wai KT, et al. Eco-biosocial determinants of dengue vector breeding: a multicountry study in urban and periurban Asia. Bulletin of the world Health Organization. 2010;88:173-84.

18. Sommerfeld J, Kroeger A. Eco-bio-social research on dengue in Asia: a multicountry study on ecosystem and community-based approaches for the control of dengue vectors in urban and peri-urban Asia. Pathogens and 
global health. 2012;106(8):428-35.

19. Keating J. An investigation into the cyclical incidence of dengue fever. Social science \& medicine. 2001;53(12):1587-97.

20. Jamil B, Hasan R, Zafar A, Bewley K, Chamberlain J, Mioulet $\mathrm{V}$, et al. Dengue virus serotype 3, Karachi, Pakistan. Emerging infectious diseases. 2007;13(1):182.

21. Khan E, Hasan R, Mehraj V, Nasir A, Siddiqui J, Hewson R. Co-circulations of two genotypes of dengue virus in 2006 out-break of dengue hemorrhagic fever in Karachi, Pakistan. Journal of Clinical Virology. 2008;43(2):176-9.

22. Rowley WA, Graham CL. The effect of temperature and relative humidity on the flight performance of female Aedes aegypti. Journal of Insect Physiology. 1968;14(9):1251-7.

23. Ali A, ur Rehman H, Nisar M, Rafique S, Ali $S$, Hussain $A$, et al. Seroepidemiology of dengue fever in Khyber Pakhtunkhawa, Pakistan. International journal of infectious diseases. 2013;17(7):e518-e23.

24. Chuang VW, Wong T, Leung Y, Ma ES, Law Y, Tsang OT, et al. Review of dengue fever cases in Hong Kong during 1998 to 2005. Hong Kong Medical Journal. 2008;14(3):170.

25. Gupta E, Dar L, Kapoor G, Broor S. The changing epidemiology of dengue in Delhi, India. virology Journal. 2006;3(1):92.

26. McBride WJH. Evaluation of dengue NS1 test kits for the diagnosis of dengue fever. Diagnostic microbiology and infectious disease. 2009;64(1):31-6.

27. Duyen HT, Ngoc TV, Ha DT, Hang VT, Kieu NT, Young PR, et al. Kinetics of plasma viremia and soluble nonstructural protein 1 concentrations in dengue: differential effects according to serotype and immune status. Journal of Infectious Diseases. 2011;203(9):1292-300.

28. Laredo-Tiscareño S, Machain-Williams C, Rodríguez-Pérez MA, Garza-Hernandez JA, Doria-Cobos GL, Cetina-Trejo RC, et al. Arbovirus Surveillance near the Mexico-US Border: Isolation and Sequence Analysis of Chikungunya Virus from Patients with Denguelike Symptoms in Reynosa, Tamaulipas. The American journal of tropical medicine and hygiene. 2018;99(1):191-4.

29. Fuchs I, Bin H, Schlezinger S, Schwartz E. NS1 antigen testing for the diagnosis of dengue in returned Israeli travelers. Journal of medical virology. 2014;86(12):2005-10. 